\title{
A case of Kingella kingae endocarditis complicated by native mitral valve
}

\section{rupture}

Mohammad Bagherirad, Damoon Entesari-Tatafi, Sam Mirzaee, Allan Appelbe, Chenghon Yap, Eugene Athan

Barwon Health - The Geelong Hospital, Departments of Infectious Diseases, General Medicine, Cardiology

\section{and Cardiothoracic Surgery}

\section{CASE REPORT}

Bagherirad MA, Entesari-Tatafi D, Mirzaee S, Appelbe A, Yap C, Athan E. A case of Kingella kingae endocarditis complicated by native mitral valve rupture. AMJ 2013, 6, 4, 172-174. http://doi.org/10.21767/AMJ.2013.1577

Corresponding Author:

Damoon Entesari-Tatafi

Barwon Health - The Geelong Hospital

Bellerine Street Geelong

Email: dentej2@hotmail.com

\section{Abstract}

We report a case of Kingella kingae endocarditis in a patient with a history of recent respiratory tract infection and dental extraction. This case is remarkable for embolic and vasculitic phenomena in association with a large valve vegetation and valve perforation. Kingella kingae is an organism known to cause endocarditis, however early major complications are uncommon. Our case of Kingella endocarditis behaved in a virulent fashion necessitating a combined approach of intravenous antibiotic therapy and a valve replacement. It highlights the importance of expedited investigation for endocarditis in patients with Kingella bacteraemia.

Key Words

Kingella kingae; Endocarditis; Mitral Valve Rupture.

\section{Implications for Practice}

1. Kingella kingae belongs to the HACEK (Haemophilus aphrophilus, Actinobacillus, Actinomycetem-comitans, Cardiobacterium hominis, Eikenella corrodens, and Kingella kingae) group of organisms known to cause endocarditis. These organisms were recognised to grow late in blood cultures incubated with traditional techniques, requiring longer incubation. This is no longer the case with modern culture techniques.

2. Kingella kingae is an oral commensal bacteria with considerable variability in pathogenicity mediated by incompletely delineated virulence factors. Embolic, vasculitic, and cardiac complications may occur as a result of unrecognised and untreated Kingella endocarditis.

3. Kingella bacteraemia may reflect underlying endocarditis and thus should be investigated in an expedited fashion with transthoracic echocardiogram and early transoesophageal echocardiogram.

\section{Background}

Endocarditis is a serious condition with high morbidity and mortality. It often presents as a febrile illness without a clear focus of infection. A cardiac murmur may or may not be initially present. The result of delayed or misdiagnosis can be catastrophic. The initial clinical clue to a diagnosis of endocarditis may be bacteraemia with a typical organism known to cause endocarditis, or persistent bacteraemia.

Kingella kingae belongs to the HACEK group of organisms known to cause endocarditis and is typically viewed as less virulent than many other bacteria causing endocarditis. They are also slower growing, often detected after five days of incubation with traditional blood culture techniques. As a result, Kingella bacteraemia could be previously missed because of insufficient incubation. This is no longer the case with modern techniques, with average time to bacterial growth occurring in less than five days.

The lower virulence of the organism has also meant that embolic and vasculitic phenomena are less common, as are cardiac complications such as valve perforation or conduction block. This allows non-surgical treatment of Kingella endocarditis to be the norm and shorter periods of intravenous antibiotic therapy possible. 


\section{Case details}

We report the case of a 51-year-old female diabetic patient with Kingella kingae native mitral valve endocarditis complicated by valve perforation.

The patient was admitted to hospital with a febrile illness for investigation. She described a four-day history of fever, myalgia and malaise associated with fluctuating blood glucose levels. Her recent medical history was significant for pharyngitis which resolved without antibiotics three weeks prior, and a periodontal abscess managed with dental extraction and a short course of antibiotics four months prior. Physical examination was unremarkable except for a non-blanching vasculitic right heellesion.

Patient's laboratory work-up was significant for a C-reactive protein (CRP) of $285 \mathrm{mg} / \mathrm{L}$, while chest X-ray and urine microscopy, culture and sensitivity test (MCS) were negative for a source of infection, and blood culture results were pending. On day 2 of admission, blood culture reports revealed growth of unidentified gram negative bacillus. She was initially managed with intravenous ceftriaxone. By day 4 of admission, the patient's CRP had improved to $64 \mathrm{mg} / \mathrm{L}$ and she was discharged with oral amoxicillin-clavulanic acid.

Day 2 post-discharge the patient was recalled to hospital following identification of penicillin-ensitive Kingella kingae. She denied any physical symptoms other than appearance of a new rash. Examination was significant for multiple nontender blanching maculopapular lesions localising to the arms and to a lesser extent the legs. A pansystolic murmur in the mitral region was also identified without signs of heart failure. The patient was suspected of having Kingella endocarditis with a vasculitic reaction. She was recommenced on intravenous ceftriaxone and further blood cultures were obtained.

Transoesophageal echocardiogram (see Figure 1) revealed a 20x25 mm mitral valve vegetation with posterior leaflet perforation. Preoperative MRI brain revealed a clinically silent $6 \mathrm{~mm}$ right sided cerebellar infarct. Multidisciplinary assessment including the neurology team deemed the risk of delayed intervention greater than the risk of bleeding at surgery. A unanimous decision was made to proceed to surgery.

The operation was uncomplicated with insertion of a mechanical mitral valve. Operative tissue sample confirmed a $25 \mathrm{~mm}$ mitral valve vegetation with posterior leaflet perforation. Further blood cultures and operative valve culture were negative. Post-operative recovery was uncomplicated and the patient was commenced on therapeutic anticoagulation with enoxaparin then transitioned to warfarin. The patient was discharged to Hospital In The Home (HITH) to complete a total six-week course of intravenous ceftriaxone.

\section{Figure 1: Photo and video of transoesophageal echocardiogram}

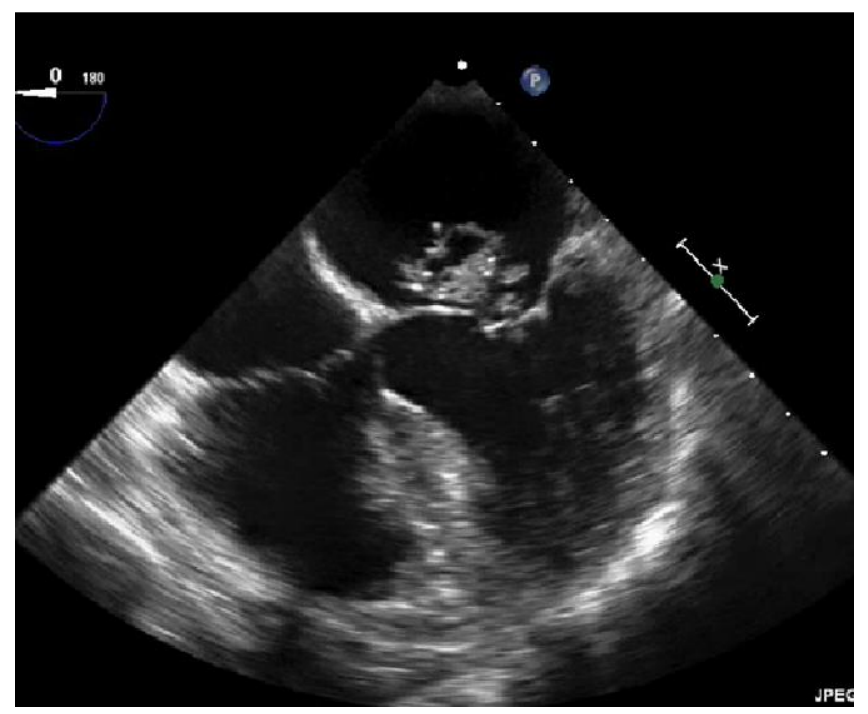

The patient was followed up weekly until treatment completion and then at two weeks post treatment in the infectious diseases clinic. Her recovery was complete and without any complication.

\section{Discussion}

Kingella kingae belongs to the HACEK group of organisms known to cause endocarditis. ${ }^{1}$ At least 25 cases of Kingella endocarditis have been reported. ${ }^{2}$ This group of bacteria are commensals of the human respiratory tract.

Little is known about the pathogenesis of Kingella infections. Epidemiologically, approximately $90 \%$ of invasive disease occurs in children. ${ }^{3}$ Considerable variability has been found in RTX toxin production, a potential virulence factor, between strains of Kingella. ${ }^{4,5}$ Kingella endocarditis usually occurs in prosthetic and or diseased valves. Our case demonstrates that Kingella endocarditis may involve healthy native valves and may result in cardiac, embolic and vasculitic complications.

Given the serious nature of Kingella endocarditis and the infrequent identification of an alternative source for Kingella bacteraemia, we recommend that all patients with Kingella bacteraemia be carefully evaluated for the presence of endocarditis. ${ }^{1}$ We also recommend early appropriate intravenous antibiotic therapy guided by 
microbiological sensitivities tests and consideration of surgical review in patients with clinically aggressive disease.

\section{References}

1. Mandell GM, Bennett JE, Dolin R. Mandell, Douglas, and Bennett's Principles \& Practice of Infectious Diseases, $7^{\text {th }}$ Edition 2010. Churchill Livingstone: USA. p.1084.

2. Jenny DB, Letendre PW, Iverson G. Endocarditis due to Kingella species. Rev Infect Dis. 1988 SepOct;10(5):1065-6.

3. Slonim A, Steiner M, Yagupsky P: Immune response to invasive Kingella kingae infections, age-related incidence of disease, and levels of antibody to outer-membrane proteins. Clin Infect Dis. 2003 Aug 15;37(4):521-7.

4. Kiang KM, Ogunmodede F, Juni BA, Boxrud DJ, Glennen A, Bartkus JM, Cebelinski EA, Harriman K, Koop S, Faville R, Danila R, Lynfield R. Outbreak of osteomyelitis/septic arthritis by Kingella among child care center attendees. Pediatrics. 2005 Aug;116(2):e206-13.

5. Kehl-Fie TE, St. Geme 3rd JW. Identification and characterization of an RTX toxin in the emerging pathogen Kingella kingae. J Bacteriol. 2007 Jan;189(2):430-6.

\section{PEER REVIEW}

Not commissioned. Externally peer reviewed.

\section{CONFLICTS OF INTEREST}

The authors declare that they have no competing interests

\section{PATIENT CONSENT}

The authors, Mohammad Bagherirad and Damoon EntesariTatafi, declare that:

1. They have obtained written, informed consent for the publication of the details relating to the patient(s) in this report.

2. All possible steps have been taken to safeguard the identity of the patient(s).

3. This submission is compliant with the requirements of local research ethics committees. 